\title{
The Simulation Analysis of Nonlinear for a Power Amplifier with Memory Effects
}

\author{
Lv. Jinqiu \\ College of Electronic and Electrical Engineering, Shanghai University of Engineering Science, Shanghai, 201620 China \\ Email: weiganglvjinqiu@ outlook.com \\ You. Xiaoming \\ College of Electronic and Electrical Engineering, Shanghai University of Engineering Science, Shanghai, 201620 China \\ Email: yxm6301@163.com

\section{Liu. Sheng} \\ College of Management, Shanghai University of Engineering Science, Shanghai, 201620 China \\ Email: 1s6601@163.com
}

\begin{abstract}
For the nonlinear distortion problem of current power amplifiers (PAs) with memory effects, we use goal programming to present a memoryless predistorter matrix model based on limiting baseband predistortion technique, and the normalized mean squared error (NMSE) is limited in a satisfactory range while the output power is maximum. Then we propose a nonlinear power amplifier with memory effects based on back propagation neural network (BPNN) with three tapped delay nodes and six single hidden layer nodes, which is single input - dual output. Simulation results show that the method proposed in this paper makes the experimental precision higher. Further, the linearization effect of power amplifiers becomes better.
\end{abstract}

Index Terms - Power Amplifier, Pred istortion Technique, Goal Programming, Tapped Delay, Back Propagation Neural Networks

\section{INTRODUCTION}

Nonlinear power amplifiers(PAs) for signals near the saturation region will have a serious spectral regrowth which causes adjacent channel interference and in-band distortion which degrades the bit-error rate(BER) performance. To ensure the efficiency and the correct signal transmitting of PAs at the same time, a PA linearization technique should be adopted. Although the transfer function of PAs is linear, its dynamic characteristics also cause a distortion called linear distortion which is easy to compensate by linear filter. However, the nonlinear distortion is inherent in the active device. The even terms in the polynomial fitting will introduce integer multiples of the frequency components and the nonlinear distortion which is called harmonic distortion, and the odd items will affect the baseband output of system. In the wireless communication system, a strict band-pass filter can filter the harmonic distortion completely; therefore it's only need to consider the odd items nonlinear distortion of PAs.

Researches on the PA memory effect is the basis of numerical analysis of PA working condition, so many scholars have worked in this area and made a lot of achievements. S. Boumaiza and F.M Ghannouchi established thermal memory effects modeling in PAs, and discussed how to compensate it in predistortion linearizres[1]. Since the number of parameters in general form Volterra series model will increase rapidly with the system order and memory depth growing which results in a large amount of computing of parameter identification, its adhibitions are limited to low-level weak memory nonlinear systems. $\mathrm{H}$. $\mathrm{Ku}$ et al. proposed a behavioral model based on the previously developed memory polynomial model [2]. It is actually a simplified form of the Volterra series model, due to the number of parameters which is far less than the number in the same order and memory depth Volterra series model, the computationally intensive of model identification is reduced greatly. In practical applications, the predistortion technology based on Volterra series is difficult to realize the self-adaption and track nonlinear drift of PAs, so predistortion is not ideal [9]. Zhong Peilin et al. used a compressing quantization method to design a non-iterative look-up table predistortion method based on waveform training, which effectively improved the power spectrum of the signal and the bit error rate performance of system, and minished the intermodulated power of signal[3]. Lv Xiu Jie et al. studied the realization form of linear subsystem in Hammerstein predistorter and the predistortion structure for identifying the predistorter parameters with the high-efficient lease square algorithm. The Hammerstein predistorter can efficiently compensate the nonlinear distortion of PAs with memory effects[4]. Zhan Peng et al. proposed the single-feedback predistortion method based on memory polynomial, which can avoid the IQ imbalance errors caused by the down-conversion circuit, reduce the costs and improve the linearization performance of digital predistortion[5]. Li Bo et al. presented a novel fractional order memory polynomial predistorter and the corresponding coefficient estimation algorith $\mathrm{m}[6]$. Yang You Fu et al. proposed an open-loop memory polyno mial 
pre-distorter based on look-up table, which can effectively compensate memory nonlinearity distortion of system due to high-power amplifier, and dramatically improve the system BER performance[7]. However, when a lookup table is used to indicate the inverse character of the power amplifier, a more complex and larger one will be required, which makes convergence of the table become very slow. N. Naskas et al. proposed a neural-network-based adaptive baseband predistortion method for RF PAs, which can achieve a significant linearity improvement that reaches $25 \mathrm{~dB}$ [8]. Yang Zhao, Zhang Qin, Xia Gaofeng, Liu Jiong proposed a digital predistortion linearization algorithm using multi-stage look-up-table predistorter with optimal linear weighted delay for memory power amplifiers, and the new algorith $\mathrm{m}$ achieved better linearization results validated by hardware tests[9]. Hosein Miar-Naimi and Hamid Rahimpour analyzed the effects of phase and gain imbalances between two signal branches in linear amp lification with nonlinear components transmitters and presented a new algorithm whose advantages are zero residual error and fast convergence time[10]. Mei Yen Cheong et al. proposed novel direct and indirect learning predistorters (PDs) that employ a new baseband simplicial canonical piecewise linear (SCPWL) function. The performance of the proposed PDs is easily controlled by varying the number of segments of the SCPWL function[11]. Felice Francesco Tafuri, Cataldo Guaragnella, Marco Fiore, and Torben Larsen proposed a linearization by means of a new type of digital predistorter, defined directly in the I-Q domain. The enhancement of this model with additiona I-Q terms can guarantee a more versatile compensation when the distortion comes from the joint contribution of the PA and the quadrature modulator[12]. Oishi, Y. et al. described a method to design a predistorter for a power amplifier with a memory effect by using nonlinear parameters extracted from measured intermodulation distortion for a GaN-FET amplifier. And they confirmed that a fifth-order predistorter was effective up to a higher power level[13]. Farouk Mkadem et al. expounded a complexity-reduced Volterra series model for radio frequency power amplifier(PA) behavioral modeling and digital predistortion(DPD) by analyzing the memory effect mechanisms of the PA[14].

The traditional way to identify predistortion based on adaptive networks is to use a training sequence to extract the PA model. Adjusting the appropriate training signal range can make power range covered by the entire gain characteristic range of PAs, which is from linear amplification to begin to distortion to complete saturation[15]. When the training signal is output to the amplifier, the obtained feedback signal is easily to correspond to the original training sequences synchronously, which greatly simplifies the problem. The synchronization technology's functional circuit is complex and it also needs to solve the problem of loop delay. Although the adaptive LMS algorithm based on Newton iteration can obtain a high precision solution, while reducing the time complexity, the in itial estimation problem of matrix sequences has a great impact on convergence of the algorith $\mathrm{m}[16]$. At first, this paper uses limiting baseband predistortion technology to establish the fitting polynomial of nonlinear Pas and gives memoryless predistorter's parameters based on objective programming algorithm of matrix analysis. The obtained satisfactory solution of predistorter does not depend on the specific characteristics of the nonlinear. Secondly, this paper researches the BPNN model accuracy of PAs and the relationship between generalization ability and the number of training samples and hidden layer neurons. Also it establishes a tapped delay BPNN model for the distortion characteristics of PAs with memory nonlinearity. Simulation results show that this model fits the nonlinear characteristics of PAs with memory precisely.

This paper is organized as follows. In Section 2 we present the memoryless effect predistortion algorithms and obtain satisfactory solution of predistorter which does not depend on the specific nonlinear characteristics. In Section 3 we establish the nonlinear PA model with memory effects based on BPNN with tapped delay nodes. In the next section we illustrate our simulation and analysis. Section 5 makes a conclusion for this paper.

\section{THE MEMORYLESS EFFECT PREDISTORTION ALGORITHMS BASED ON ANALYSIS OF PHASE ANGLE AND AMPLITUDE}

\section{A. The mathematical model of nonlinear PAs}

Set as the input signal of PAs, where $r_{n} 、 \theta_{n}$ represent the amplitude and phase angle respectively. Then the corresponding output signal amplitude is as follows:

$$
\begin{aligned}
\hat{z}\left(r_{n}\right)= & \left.G^{T} R_{h}\right|_{x=r_{n}} \\
= & h(1) x(t)+h(2) x(t)^{3} \\
& +h(3) x(t)^{5}+h(4) x(t)^{7}
\end{aligned}
$$

where $\hat{z}\left(r_{n}\right) n=1,2, \cdots, 1000$ is the output signal amplitude in simulations , $G=[h(1), h(2), h(3), h(4)]^{T}$ is fitting polynomial coefficient vector of PAs characteristics, $G()$ is the nonlinear characteristic function of PAs, and $R_{h}=\left[x(t), x(t)^{3}, x(t)^{5}, x(t)^{7}\right]^{T}$ is the orthogonalbasis vectors.

The output signal AM and PM of PAs are mainly determined by the amplitude of the input signal and almost independent of the phase of the input signal[17]. So the memoryless PA will only produce amplitude-toamplitude modulation (AM-AM) distortion. To avoid Runge phenomenon, we use seven times polynomials, and polynomial contains only odd items. 


\section{B. Predistorter goal programming model based on matrix} analysis

$$
\text { Set } J\left(f_{i j}\right)=\sum_{n=1}^{1000}\left|G \bullet\left(f_{i j}\right)_{4 \times 4} R_{h}\right|_{r_{n}}-\left.g r_{n}\right|^{2}
$$

Where $F(x(t))=\left(f_{i j}\right)_{4 \times 4} R_{h}$ is the characteristic function of a predistorter, which produces the corrective action in the opposite direction for the nonlinear compensate of PAs and is fitted by polynomial matrix, and constant $\mathrm{g}$ is the ideal amplitude magnification of PAs. The total effect makes the overall input-output characteristic linearized, and the output power be fully utilized.

(NMSE) can be described by

$$
N M S E=10 \lg \frac{\sum_{n=1}^{N}|z(n)-\hat{z}(n)|^{2}}{\sum_{n=1}^{N}|z(n)|^{2}}
$$

For the minimum value of NMSE, as well as the best linearization degree of PAs, we obtain equations as follows:

$$
\frac{\partial J\left(f_{i j}\right)}{\partial f_{i j}}=0 \quad, \quad i, j=1,2, \cdots, 4
$$

Using Mathametica 7.0.0 to solve (4), we obtain the following equations:

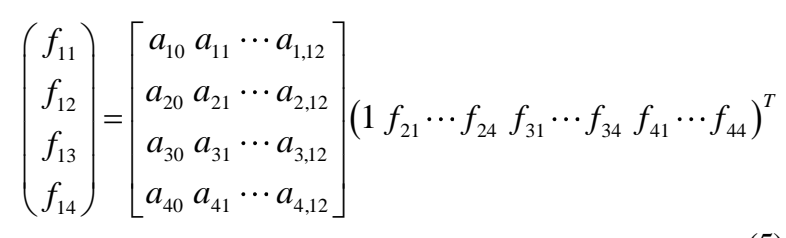

In order to give full play to the role of PAs, making the average output power maximize must be considered in building the predistortion processing model. This paper establishes an objective programming model which consists of NMSE, limiting output amplitude, maximizing power and other constraints after determining the nonlinear characteristics of PAs.

Set $f_{21}, \cdots, f_{24}, f_{31}, \cdots, f_{34}, f_{41}, \cdots, f_{44}$ as the decision variables of the objective programming.

$$
\left\{\begin{array}{c}
N M S E+d_{1}^{-}-d_{1}^{+}=c_{1} \\
\bar{P}+d_{2}^{-}-d_{2}^{+}=c_{2} \\
\left|x_{i j}\right| \leq M, i, j=1, \cdots, 4 \\
d_{l}^{-}, d_{l}^{+} \geq 0, l=1,2
\end{array}\right.
$$$$
\min Z=p_{1} d_{1}^{+}+p_{2} d_{2}^{-}
$$

Since the oversized input amplitude will cause an overflow saturation of PAs, it's need to limit the predistortion output amplitude to not be greater than the maximum of the given input amplitude of PAs. In (6), the bigger the signal average power after predistortion processing $\bar{P}=\left.\frac{1}{N} \sum_{n=1}^{N}\left|\left(f_{i j}\right)_{4 \times 4} R_{h}\right|_{x=x(n)}\right|^{2} \quad$ is, the better[19]. According to the average output power of PAs the average input power target can be calculated. $p_{1}=\frac{2}{3}, p_{2}=\frac{1}{3}, 16$ rigid constraints meet $|x(n)| \leq M$. The tolerance $\mathrm{M}$ of input amplitude is determined by the maximum amplitude of the input signals, i.e., $M$ is the maximu module value of elements in the matrix $\left(f_{i j}\right)$.

$$
\begin{gathered}
F\left(x\left(r_{n}\right)\right)=\left.\left(f_{i j}\right)_{i, j=1, \cdots, 4} R_{h}\right|_{r_{n}}, \\
n=1,2, \cdots, N
\end{gathered}
$$

\section{THE NONLINEAR PA MODEL WITH MEMORY EFFECT S BASED ON BPNN WITH TAPPED DELAY NODES}

A basic BPNN is composed of the input output layer which consists of input output nodes and tapped nodes, and the hidden layer which consists of hidden layer nodes. The larger the size of the network is, the stronger capability of network function mapping is, the stronger the memory of the training samples is, while the worse the fault tolerance of a new sample is as well as, the worse the generalization capability of the network is [18].

$$
x_{-} n=\frac{x-\min (x)}{\max (x)-\min (x)}
$$

where $\mathrm{x}$ is the input signal amplitude of, $x_{-} n$ is the input signal amplitude after normalization, $\min (x)$ is the minimum value of the input signal amplitude, $\max (x)$ is the maximum value of the input signal amplitude.

In the BPNN model, the input node is the input amplitude of PAs, and the output node is the output amplitude and output phase angle of PAs.

If BPNN needs to reflect the me mory characteristics of PAs, it must add a delay link at the input node, i.e., add tapped delay nodes, and the number of nodes is the memory depth m of PAs. By Matlab simulation analysis, the BPNN fitting effect becomes more and more ideal with tapped delay nodes increasing, but the algorithm running time is getting longer and longer, and work efficiency is lower and lower. Taken together, the best number of tapped nodes in this model is 3 .

In BPNN, if the numbers of hidden layer nodes are different, fitting results obtained are also different. When the number of hidden layer nodes is 6 , the fitting curve can reflect the orig inal PA nonlinear characteristics well. The different excitation functions will also affect the 
fitting effect of neural network model. Frequently-used excitation functions are logsig, pureline, poslin and tanhsig[20]. In this paper, we use sigmoid excitation function which can reflect the nonlinear characteristic of PAs well. Nonlinear model for a single input - dual output neural network model and contain six single hidden layer nodes.

The solving steps of BPNN model are as follows:

(1) Initialization: Give random nu mbers between -1 to +1 to the each connection weight $\left\{\omega_{i j}\right\},\left\{v_{j t}\right\}$ and threshold $\left\{\theta_{j}\right\},\left\{\gamma_{t}\right\}$. Ain: input signal amplitude; Aout: output signal amplitude; $\Phi_{\text {out }}$ : output signal phase angle; $\omega_{i j}$ : connection weight between input node $\mathrm{i}$ and hidden layer node $\mathrm{j} ; v_{j t}$ : connection weight between hidden layer node $\mathrm{j}$ and output node $\mathrm{t} ; \theta_{j}$ : output bias of hidden layer node $\mathrm{j} ; \gamma_{t}$ : output bias of output layer node t.

(2) Use the input sample $A^{k}=\left(a_{1}, \cdots, a_{n}\right)$, the connection weights $\left\{\omega_{i j}\right\}$ and thresholds $\left\{\theta_{j}\right\}$ to calculate the input $\left\{s_{j}\right\}$ and output $\left\{b_{j}\right\}$ of each unit of the hidden layer.

$s_{j}=\sum_{i=1}^{n} \omega_{i j} a_{j}-\theta_{j}$,

$b_{j}=f\left(s_{j}\right)=\frac{1}{1+e^{-s_{j}}}$,

$j=1,2, \cdots, p$

(3) Calculate the input $\left\{L_{t}\right\}$ of each unit of the output layer, and calculate the response $\left\{C_{t}\right\}$ of each unit of the hidden layer by Sigmoid function.

$L_{t}=\sum_{j=1}^{p} v_{j t} b_{j}-\gamma_{t}$,

$C_{t}=f\left(L_{t}\right)=\frac{1}{1+e^{-L_{t}}}$,

$t=1,2, \cdots, q$

(4) Use $\left\{C_{t}\right\}$ and hope output samples $Y^{k}=\left(y_{1}, \cdots, y_{n}\right)$ to calculate the generalization error $\left\{d_{t}^{k}\right\}$ of each unit of the output layer, and correct connection weights $\left\{v_{j t}\right\}$ and thresholds $\left\{\gamma_{t}\right\}$ by $\left\{b_{j}\right\}$.

$d_{t}^{k}=\left(Y_{t}^{k}-C_{t}^{k}\right) C_{t}\left(1-C_{t}\right), k=1, \cdots, m$

$v_{j t}(N+1)=v_{j t}(N)+\beta d_{t}^{k} b_{j}$

$$
\gamma_{j}(N+1)=\gamma_{j}(N)+\beta d_{t}^{k}, \quad i=1, \cdots, n
$$

(5) Use $\left\{v_{j t}\right\},\left\{d_{t}^{k}\right\}$ and $\left\{b_{j}\right\}$ to calculate the generalization error $\left\{e_{j}^{k}\right\}$ of each unit of the hidden layer and $A^{k}$, and correct $\left\{\omega_{i j}\right\}$ by $\left\{\theta_{j}\right\}$.

$e_{j}^{k}=\left(\sum_{t=1}^{q} d_{t}\right)\left(\gamma_{j t}\right) b_{j}\left(1-b_{j}\right), j=1,2, \cdots, p$,

$\omega_{i j}(N+1)=\omega_{i j}(N)+\beta e_{j}^{k} a_{i}$

$\theta_{j}(N+1)=\theta_{j}(N)+\beta e_{j}^{k}, i=1, \cdots, n$

(6) Randomly select the next learning mode pair to the network, and return to (2) until all learning mode pairs have been trained completely.

(7) Randomly reselect a learning mode pair from the $n$ learning mode pairs, and return to (2) until the BPNN global error function $\mathrm{E}$ is smaller than a preset value, i.e., the network converges. $\mathrm{E}$ is calculated by (13);

$$
E=\sum_{k=1}^{m} E_{k}=\sum_{k=1}^{m} \sum_{t=1}^{q} \frac{\left(y_{t}^{k}-C_{t}\right)^{2}}{2}
$$

\section{SimULATION ANALYSE}

Running the Matlab procedure, we obtain Fig. 1 and Fig. 2. While fitting (1), we obtain vector $G=[3.0279,-0.6683,-1.4093,0.9523]^{T}$ and Fig.3. So the amplitude polynomial of PAs is following:

$$
\begin{aligned}
G(x)= & 3.0279 x-0.6683 x^{3} \\
& -1.4093 x^{5}+0.9523 x^{7}
\end{aligned}
$$

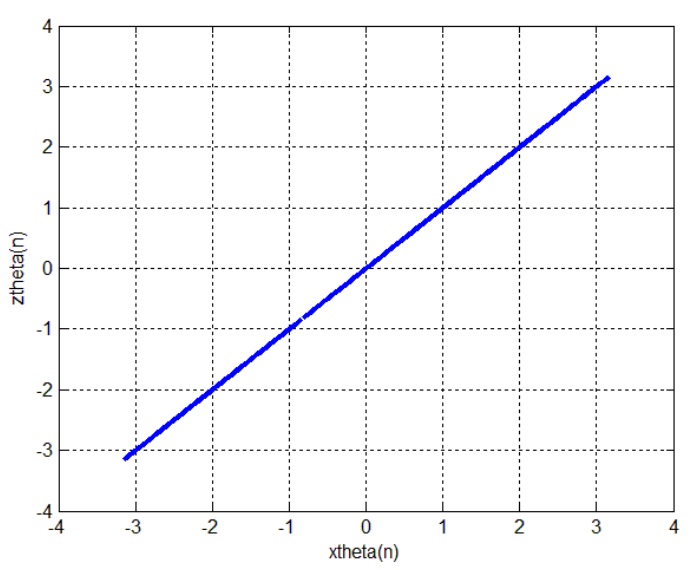

Fig. 1. Memoryless PA input / output phase angle scatter 


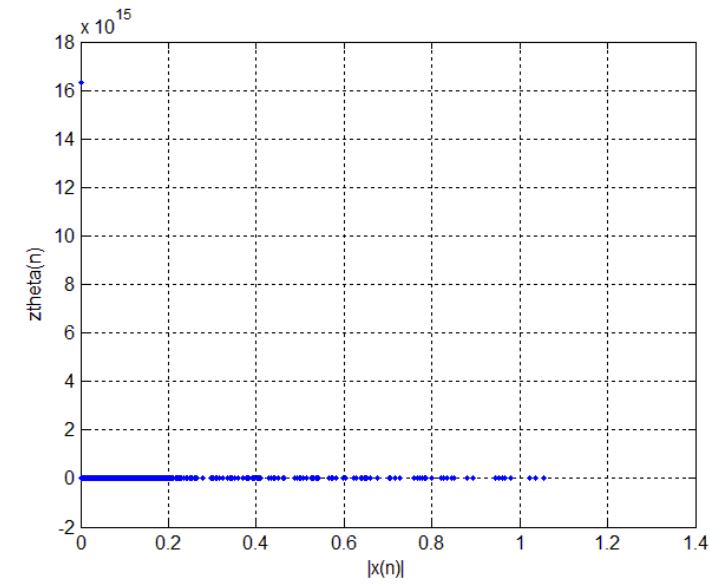

Fig. 2. Memoryless PA input amplitude / output phase angle scatter

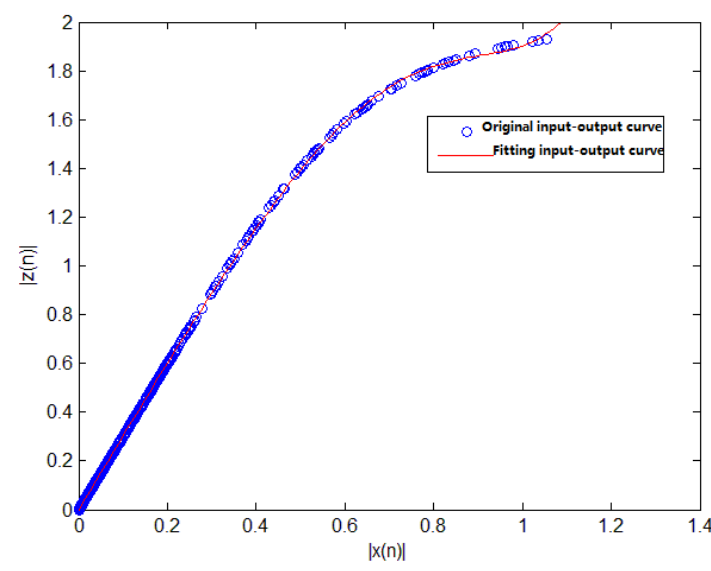

Fig. 3. Memoryless PA input /output polynomial fitting

Table 1. Memoryless predistorter simulation results

\begin{tabular}{|c|c|c|c|c|c|c|}
\hline & Matrix & $\left.f_{i j}\right)$ & & $M$ & $N M S E$ & $\bar{P}$ \\
\hline 0.7241 & 0.0649 & 0.0743 & 0.0743 & \multirow{4}{*}{1.1168} & \multirow{4}{*}{-10.2629} & \multirow{4}{*}{0.1851} \\
\hline 0.2000 & 0.3000 & 0.2000 & 0.2000 & & & \\
\hline 0.3000 & 0.2000 & 0.2000 & 0.200 & & & \\
\hline 0.2000 & 0.3000 & 0.2000 & 0.200 & & & \\
\hline 0.7241 & 0.0743 & 0.0184 & 0.0743 & \multirow{4}{*}{1.1301} & \multirow{4}{*}{-10.2628} & \multirow{4}{*}{0.1808} \\
\hline 0.2000 & 0.2000 & 0.3000 & 0.2000 & & & \\
\hline 0.3000 & 0.2000 & 0.1000 & 0.2000 & & & \\
\hline 0.2000 & 0.2000 & 0.3000 & 0.2000 & & & \\
\hline 0.6776 & 0.0523 & 0.0743 & 0.0523 & \multirow{4}{*}{0.9993} & \multirow{4}{*}{-10.2640} & \multirow{4}{*}{0.160} \\
\hline 0.2000 & 0.1000 & 0.2000 & 0.1000 & & & \\
\hline 0.2000 & 0.2000 & 0.2000 & 0.2000 & & & \\
\hline 0.2000 & 0.2000 & 0.2000 & 0.2000 & & & \\
\hline 0.6310 & 0.1058 & 0.0523 & 0.0057 & \multirow{4}{*}{0.8817} & \multirow{4}{*}{-10.2623} & \multirow{4}{*}{0.1435} \\
\hline 0.2000 & 0.2000 & 0.1000 & 0.1000 & & & \\
\hline 0.1000 & 0.2000 & 0.2000 & 0.1000 & & & \\
\hline 0.2000 & 0.1000 & 0.2000 & 0.2000 & & & \\
\hline 0.7241 & 0.1279 & 0.0208 & 0.0988 & \multirow{4}{*}{1.2505} & \multirow{4}{*}{-10.2627} & \multirow{4}{*}{0.1809} \\
\hline 0.2000 & 0.3000 & 0.1000 & 0.1000 & & & \\
\hline 0.300 & 0.200 & 0.200 & 0.300 & & & \\
\hline 0.200 & 0.100 & 0.300 & 0.200 & & & \\
\hline
\end{tabular}

Using objective programming, calling Matlab procedure, then we calculate the corresponding $\mathrm{M}$, NMSE and $\bar{P}$. In table 1 , the best group is $\bar{P}=0.1851$, $\mathrm{NMSE}=-10.2629$.

Using BPNN to solve nonlinear model of PAs with memory, we obtain results in Fig. 4.
As shown in Fig 1, the red fitting curve is almost covered by the original input data, i.e., BPNN output results reflect the nonlinear characteristic of PAs effectively. Since the established neural network model is single input and dual output, i.e., the original output signal is divided into amplitude and phase, the evaluation 
of this model should be divided into two parts. In this model, amplitude normalized mean squared error ANMSE $=-17.135$; phase normalized mean squared PNMSE $=-13.324$; so we can conclude that the model can well fit the nonlinear characteristics of PAs with memory.

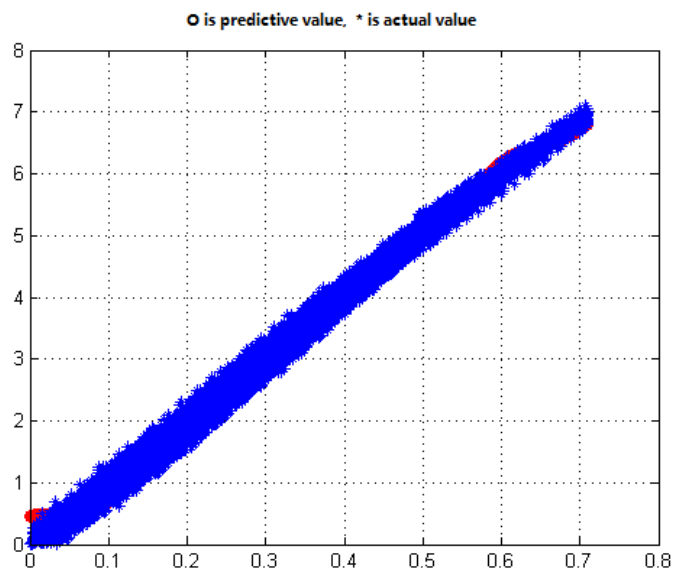

Fig. 4. BPNN output amplitude

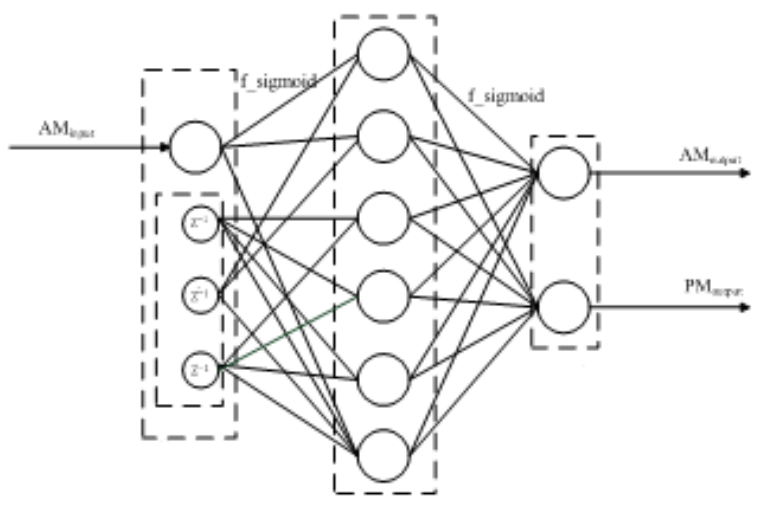

Fig. 5. BPNN model

\section{CONCLUSION}

In this brief, a memoryless nonlinear PA predistortion model has been presented which can consider the efficiency nonlinear characteristics of PAs reasonably. Simulation results show that when the maximum average output power of the predistorter reaches 0.1851 , the normalized mean square error is only -10.2629. In addition, a predistortion scheme of memory BPNN with tapped delay nodes has been proposed, which can eliminate the in-band distortion results from the memory nonlinear PA. In future work, we will consider the predistorter model with me mory PAs, which can not only improve efficiency of PAs, but also reduce spectral regrowth.

\section{ACKNOWLEDGMENT}

I would like to thank the anonymous reviewers for their careful reading of this paper and for their helpful comments.
This work was supported in part by the Innovation Program of Shanghai Municipal Education Commission (Grant No.12ZZ185), Natural Science Foundation of China (Grant No.61075115), Foundation of No.XKCZ1212.

\section{REFERENCES}

[1] S. Boumaiza and F.M. Ghannouchi, "Thermal Memory Effects Modeling and Compensation in RF Power Amplifiers and Predistortion Linearizers", IEEE Trans.on Microwave Theory and Techniques, vol. 51, no. 12, pp. 2427-2433, 2003.

[2] H. Ku and J.S. Kenney, "Behavioral Modeling of Nonlinear RF Power Amplifiers Considering Memory Effects", IEEE Trans. on Microwave Theory and Techniques, vol.51, no. 12, pp. 2495-2503, 2003.

[3] Peilin Zhong et.al. "Predistorion Algorithm Based on Compressing Quantization for Nonsinusoidal Orthogonal Modulation Signal in Time-domain", Journal of Electronics \& Information Technology, vol. 35, no. 3, pp. 658-663, 2013.

[4] Xiujie $\mathrm{Si}$ et.al. "A Novel Adaptive Structure for Hammerstein Predistorter", Journal of Electronics \& Information Technology, vol. 33, no. 6, pp. 1345-1349, 2011.

[5] Peng Zhan et.al. "Singal Feedback Predistortion Linearization Method for RF Power Amplifier", Journal of Electronics \& Information Technology, vol. 33, no. 8, pp. 2023-2027, 2011.

[6] Bo Li et.al. "A Novel Fractional Order Memory Polynomial Predistoter", Journal of Electronics \& Information Technology, vol. 31, no. 8, pp.961-964, 2009.

[7] Youfu Yang et.al. "Open-loop memory polynomial predistorter based on look-up table", Journal of Beijing University of Aeronautics and Astronautics, vol. 38, no. 3, pp. 400-404, 2012.

[8] N.Naskas and Y.Papananos. "Neural-Network-Based Adaptive Baseband Predistortion Method for RF Power Amplifiers", IEEE Trans.on Circuits and Systems, vol. 51, no. 11, pp. 619-623, 2004.

[9] Yang Zhao, Zhang Qin, Xia Gaofeng and Liu Jiong, "PA Linearization Using Multi-stage Look-Up-Table Predistorter with Optimal Linear Weighted Delay", International Conference on Signal Processing Proceedings, vol. 1, pp.47-51, 2012.

[10] Hosein Miar-Naimi and Hamid Rahimpour, "Mismatch Calibration in LINC Power Amplifiers Using Modified Gradient Algorithm", I. J. Intelligent Systems and Applications, vol. 10, pp. 59-67, 2013.

[11] Mei Yen Cheong, Stefan Werner, Marcelo J. Bruno, Jose L. Figueroa, "Adaptive Piecewise Linear Predistorters for Nonlinear Power Amplifiers With Memory", IEEE Transactions on Circuits and Systems, vol. 59, no. 7, pp. 1519-1532, 2012.

[12] Felice Francesco Tafuri, Cataldo Guaragnella, Marco Fiore, and Torben Larsen, "Linearization of RF Power Amplifiers Using an Enhanced Memory Polynomial Predistorter", NORCHIP 2012 Conference.

[13] Oishi, Y., Kimura, S., Takago, D., Daido, Y., Araki, K., Fukuda, E., Takano, T. "Design of Predistorter Using Measured Nonlinear Characteristics of Power Amplifier with Memory Effect", IEEE 75th Vehicular Technology Conference, VTC Spring 2012 - Proceedings.

[14] Farouk Mkadem, Marie Claude Fares, Slim Boumaiza and John Wood, "Complexity-reduced Volterra series model 
for power amplifier digital predistortion", Analog Integrated Circuits and Signal Processing, 2014.

[15] Lei Ding, Tong Zhou, Dennis R. Morgan et al. “A Robust Digital Baseband Predistorter Constructed Using Memory Polynomials", IEEE Transactions on Communications, vol. 52, no. 1, pp. 159-165, 2004.

[16] Hua Cui, "Neural Network Theory and its Applications to Digital Basedband Predistortion for Nonlinear PA with Memory", XiDian University 2008.

[17] Yeqing Qian and Tianren Yao, "Neural Network Predistortion Technique for Nonlinear Power Amplifiers with Memory", Computer Engineering and Applications, vol. 40, no. 21, pp. 100-103, 2004.

[18] Yong Wang, Xin Xiang and Kechu Yi, "Memory digital baseband predistorter based on polynomial", Journal of XiDian University, vol. 33, no. 2, pp. 223-227, 2006.

[19] Aihong Li, Shanzhu Xiao and Eryang Zhang, "A Method of Adaptive Volterra Predistortion Based on the Discrete Wavelet Transform", Signal Processing, vol. 25, no. 1, pp. 44-43, 2009.

[20] Xin Hu, Gang Wang, Zicheng Wang and Jirun Luo, "Effect on the linearity improvement of TWTA by combining RF predistortion linearizer and digital predistortion algorithm", Journal on Communications, vol. 33 , no. 7, pp. 156-163, 2102.

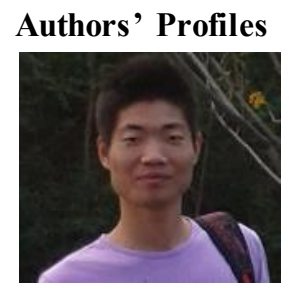

Lv.JinQiu was born in ZhenJiang, JiangSu, China in 1991. He received the Bachelor degree (2013) in Jiangsu University of science and technology, JiangSu, China. Now he is MSc student in the field of Intelligent Algorithms optimization in Shanghai University of Engineering Science. His current research interests include intelligent information processing and embedded systems.

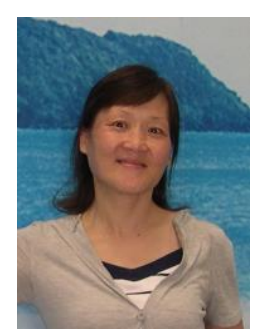

You. Xiaoming was born in 1963. She is corresponding author. She received her M.S. degree in computer science from Wuhan University in 1993, and her Ph.D. degree in computer science from East China University of Science and Technology in 2007. Her research interests include quantum-inspired evolutionary computation, distributed parallel processing and evolutionary computing. She now works in Shanghai University of Engineering Science as a professor.

Liu. Sheng was born in 1966. he received her M.S. degree in computer science from Huazhong University of Science and Technology in 1999, and his Ph.D. degree in computer science from East China University of Science and Technology in 2008. Her research interests include intelligent information processing and evolutionary computing. He now works in Shanghai University of Engineering Science as a professor.

How to cite this paper: Lv. Jinqiu, You. Xiaoming, Liu. Sheng,"The Simulation Analysis of Nonlinear for a Power Amplifier with Memory Effects", International Journal of Intelligent Systems and Applications(IJISA), vol.6, no.10, pp.20-26, 2014. DOI: $10.5815 /$ ijisa.2014.10.03 